\title{
Luminescent microscopy of the small ensembles of the CdSe nanocrystalls synthesized in the liquid crystal matrix of the cadmium octanoate
}

\author{
K.A. Magaryan ${ }^{1, *}$, I.Y. Eremchev ${ }^{2}$, K.R. Karimullin ${ }^{1,2}$, and I.A. Vasilieva ${ }^{1}$ \\ ${ }^{1}$ Moscow State Pedagogical University, 119435 Moscow, Russia \\ ${ }^{2}$ Institute for Spectroscopy RAS, 108840 Troitsk, Moscow, Russia
}

\begin{abstract}
Here we present investigation of the thin film with CdSe nanocrystals with the size $2.3 \mathrm{~nm}$ made from low-concentration toluene solution. Using fluorescent microscopy method we have found small nanocrystals ensembles up to single nanocrystals. Luminescence spectra in different parts of the test sample were registered. Analysing them we can draw a conclusion about the inhomogeneous nature of the luminescence spectra of the bulk nanocomposite material.
\end{abstract}

Combination of the unique properties of the new functional nanostructures and the unique features of the liquid crystal (LC) materials expands possibilities for their potential use significantly. Today, there are three main approaches for obtaining composites based on LC materials with nanostructures. They are doping LC matrix with nanoparticles, spraying nanostructures on the LC substrate surface and in-situ method of the direct synthesis inside the LC material [1]. To investigate spectral characteristics of the 3rd type structures we were provided with the CdSe quantum dots (QDs) grown directly in the ionic thermotropic LC matrix [2].

Polycrystalline powder with CdSe QDs was dispersed in chemically pure toluene and deposited on a substrate. After that it was placed on a precise 2D scanning stage of the fluorescence microscope-spectrometer [3]. The XY travel range was $25 \mu \mathrm{m}^{2}$ with a step of $0.5 \mu \mathrm{m}$ approximately. Luminescence spectrum was recorded on every step. All luminescence spectra were obtained at room temperature using DPSS laser diode pumping at wavelength of $405 \mathrm{~nm}$.

Inside the red frame on the Fig. 1 there are several examples of the recorded spectra of the different diffraction-limited areas on the investigated region. As one can see these curves fall in the spectral region of a broad luminescence band (purple curve), which was previously associated with the decay of excitons localized on the intrinsic defects on the QD interface [4]. The peak at wavelength of $518 \mathrm{~nm}$ is the most frequently detected. It corresponds to the interband transition characteristic for nanocrystals with a size of $2.3 \mathrm{~nm}$. The broad band in the region $575-750 \mathrm{~nm}$ is inhomogeneously broadened because it contains many wide and narrow individual lines. Some of these lines show the effect of blinking [5-9]. This blinking, as well as small FWHM (10 $\mathrm{nm}$ at the average) of these lines

${ }^{*}$ Corresponding author: ka.magaryan@phys.mpgu.edu 
indicate that the light sources are QDs with larger diameter. Apparently QDs grew to various sizes at the stage of synthesis of nanocomposite material and now it gives contribution to the bulk fluorescence spectrum.

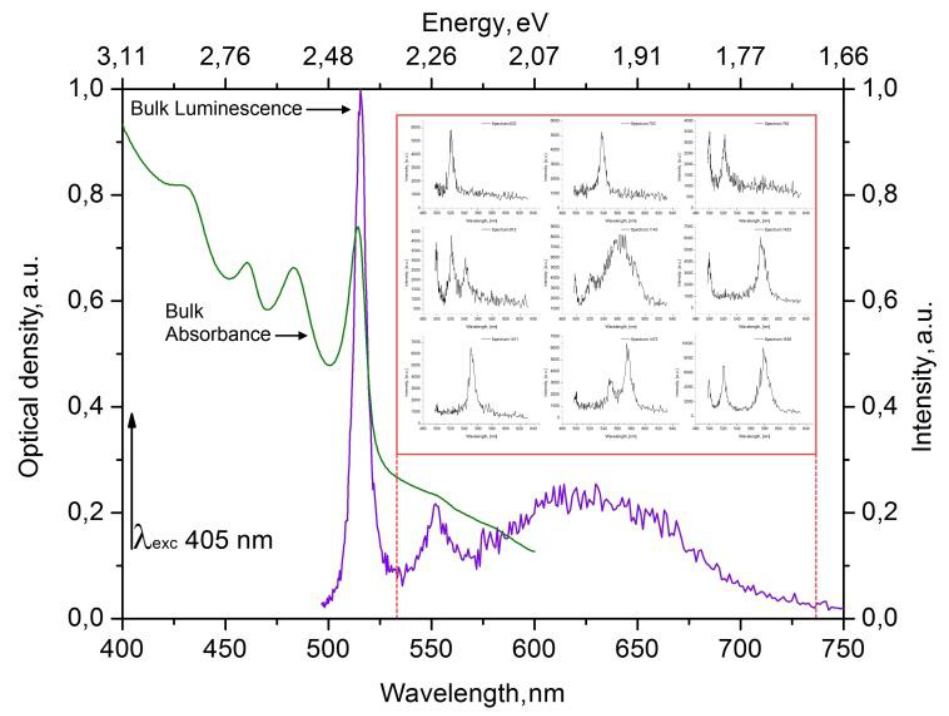

Fig. 1. Green solid curve - absorption spectrum of the toluene solution with $2.3 \mathrm{~nm}$ CdSe QDs. Purple solid curve is the fluorescence spectrum of the same solution registered from the bulk volume. Curves in the red frame show multiple luminescence spectra registered in the different parts of the thin film with CdSe QDs.

The authors are grateful to Prof. G.V. Klimusheva (Institute of Physics, National Academy of Sciences, Kyiv, Ukraine), Prof. T.A. Mirnaya (V.I. Vernadskii Institute of General and Inorganic Chemistry, National Academy of Sciences, Kyiv, Ukraine) for the opportunity to study sample with CdSe QDs, and Prof. Dr. A.V. Naumov for useful discussions.

The work is supported by the Russian Foundation for Basic Researches (project № 1532-21100-mol_a_ved). Program of Presidium RAS "Fundamental and applied problems of Photonics and physics of new optical materials". K. Magaryan acknowledges the Ministry of Education and Science of the Russian Federation (project No 14.B25.31.0007).

\section{References}

1. Li Quan, Nanoscience with Liquid Crystals (Springer, USA, 2014)

2. D. Zhulai, D. Fedorenko, A. Kovalchuk, S. Bugaychuk, G.V. Klimusheva, T.A. Mirnaya, NRL 10, 66 (2015)

3. A.V. Naumov, I.Yu. Eremchev, A.A. Gorshelev, Eur. Phys. J. D 68, 348 (2014)

4. K.A. Magaryan, M.A. Mikhailov, K.R. Karimullin, M.V. Knyazev, I.Yu. Eremchev, A.V. Naumov, I.A. Vasilieva, G.V. Klimusheva, J. Lumin. 169, 799 (2016)

5. T. Plakhotnik et al., Phys. Rev. Lett. 105, 167402 (2010)

6. M.J. Fernee et al., J. Phys. Chem. Lett. 3, 1716 (2012)

7. A.L. Shchukina, I.Yu. Eremchev, A.V. Naumov, Phys. Rev. E 92, 032102 (2015)

8. I.S.Osad'ko, I.Yu. Eremchev, A.V. Naumov, J. Phys. Chem. C 119, 22646 (2015)

9. I.Yu. Eremchev, I.S.Osad'ko, A.V. Naumov, J. Phys. Chem. C 120, 22004 (2016) 